\title{
Relevant Discussion about the Application of Molecular Imprinting Technology in Environmental Science
}

\author{
Weidong $\mathrm{Fu}$ \\ Zhejiang University of Technology, Xiaoshan, 311200, China
}

Keywords: Molecular imprinting technology, Environmental science, Application analysis

\begin{abstract}
In recent years, molecular imprinting technology rises gradually along with the development of science and opens a new perspective for the study of environmental science. In this chapter, the author introduces the principle of molecular imprinting technology briefly and explores the application of molecular imprinting in environmental science with the aim of making contributions for the development of molecular imprinting technology in our country.
\end{abstract}

\section{Introduction}

Molecular imprinting technology is a newly technology in recent years which is widely acknowledged along with the development of science and has been widely used in fields of bioscience, material science, chemistry and environmental science. In today's world, there are many reports about the molecular imprinting technology. Some foreign experts have begun the careful exploration about it and introduced it into the field of environmental science. In China, the study about the molecular imprinting technology develops gradually. This article conducts a brief exploration about molecular imprinting technology from the perspective of environmental science.

\section{The Production and Development of Molecular Imprinting Technology}

The full name of the MIT is 'Molecular Imprinting Technique'. Imprinting technology, which derives from the development of immunology, is the method which transfers the various biomacromolecules from gel to stable substance. Early in the 40s of the last century, Pauling, the Nobel Prize winner, was the first one that put forward the synthetic antibody theory modeled by antigen. ${ }^{2}$ It is considered as the initial form of the theory of molecular imprinting technology. After 10 years when Dickey raised the concept of 'molecular imprinting', the name of the molecular imprinting technology was born. However, his concept did not arouse much concern at that time. In 1972 when Wulff, a well-known professor in German reported the synthetic organic imprinting polymers, the technology became the focus gradually. Later in 1993, Professor Vlatakis from Sweden University delivered a report about the MIPs of theophylline in Nature, which helped the molecular imprinting technology infiltrate into the fields of chemistry and biology. Since then, the technology gained huge development.

At present, more than 100 social groups are working on the study of molecular imprinting technology and it covers more than 10 countries including America, Germany, Britain, China, Japan and France. In the early stage, the study of MIPs mainly focused on the fields of chiral material resolution, selective separation of substrate, chemicobiological sensor, solid phase extraction, insecticide, toxin and food groups. In recent years, the study of MIPs covers new areas. For example, it gains certain development in aspects of biomimetic sensors, the controlling of the chemical reaction process, the enrichment of the analyte in low density, the extraction of by-products and capillary electrochromatography.

\section{The Principle of Molecular Imprinting Technology}

The molecular imprinting technology mainly refers to a preparative technology which produces the polymer matched with a molecule through the joint point on the space. ${ }^{3}$ The concrete manufacturing flow is divided into 3 steps: firstly, combine the template molecule with the proper 
functional monomer in certain pore-foaming agent and form a monomer- the polymer of template molecular by relying on the covalent relationship among the functional groups. Secondly, add in the cross-linking agent when the monomer- polymer of template molecule is combined and attain the polymerization of light or heat through the effect of the initiator. Thus the formative compound and cross-linking agent will aggregate around the template molecule and the rigid polymer with high crosslinking behavior will be formed eventually; Finally, isolate the template molecule from the formative polymer so as to make it form a three-dimensional cavity which totally matches the template molecule in structure and contains the functional group which can be combined with the template molecule. The three-dimensional cavity formed under this situation possesses certain space structure and functional monomer which are determined by the quality and structure of the template molecule. Therefore, it is consistent with the template molecule.

The MIPs manufactured by the different template molecules are different, which causes the different structure and quality. One MIP can only be combined with one certain molecule, which means that MIPs are selective in the template molecule. This selectivity is some kind of 'memorability' when it is reflected on the polymer itself. That is a recognition system similar to the one possessed by nature creatures. MIPs have many advantages like strong affinity, high selectivity, strong ability to fight the severe environment, long service time and good stability, so that it can be applied in various areas.

\section{The Application of Molecule Imprinting Technology in Field of Environmental Science}

\section{The Application of Molecule Imprinting Technology in Solid Phase Extraction}

The appearance of molecules results in the rapid development of solid phase extraction. In modern technology, generally the solid phase technology which replaces the solvent extraction is adopted to conduct the bio-extraction. In the meantime, MIPs are used to enrich the targeted analyte selectively. MIPs, which is a technology with strong property, can be used in both organic solvent and water solution. Therefore, compared with other extraction technology, this technology is more advantageous. In addition, the favorable property of MIPs makes it work normally even in extreme environment. Then we know that the advantage of MIPs is beyond comparison. ${ }^{4}$ MIPs owns the characteristics of selectivity and affinity, so that it can overcome the complicated environment and inconvenient factors when it is used as the solid phase extraction to develop the biggest advantage of the sample.

MIPs technology can improve the environment's detection sensitivity to various insecticides. In environmental ministering, trace substances like various herbicides, insecticides and female hormone will be conducted the adsorption enrichment treatment and it becomes easier to detect the various doses after treatment. Therefore, we say that the MIPs technology can improve the detection sensitivity of environment. MIPs researched by scientists like Stanker can conduct the enrichment treatment toward the herbicide in the ox liver so as to improve the precision of the chromatography of the high efficiency liquid. The detection result is $5 * 10-3 \mathrm{mg} / \mathrm{L}$. Immer conducted selective enrichment toward the micro pesticide in environmental water and sediment sample by taking advantage of the terbuthylazine. Apart from the terbuthylazine, the recovery rate of all polymers is $80 \%$ and the detection result is $0.05 \sim 0.2 \mu \mathrm{g} / \mathrm{L}$.

\section{The Application of Molecule Imprinting Technology in Membrane Seperation}

Membrane separation technology of MIPs is mainly a separation technology which separates it from the substract by taking advantage of the high selectivity of a certain molecule to the MIPs membrane. MIPs membrane, which possesses strong adsorbability, have strong selectivity about the adsorption of the targeted molecule as well as high adsorption capacity. In addition, it owns large handling capacity and is easy to be magnified. Therefore, it is favored by the scientists.

The substances which applies the MIPs technology into membrane separation are amino acid and its derivative, etc. Wang produces the MIPs thin membrane of the theophylline in phase inversion. This thin membrane is, in fact, the polymer of acrylonitrile and crylic acid. After the adsorption 
experiment, the conclusion is that the adsorption capacity of the theophylline is much more greater than that of the caffeine. Kochkodam manufactures the composite membrane of the desmetryn with the method of photo-initiation and applies this method in the herbicide which contains many desmetryns. The experiment proves that it has the highest adsorption capacity toward the demetryn and it reaches $10.2 \mu \mathrm{g} / \mathrm{cm} 2$.

Advanced water treatment technology mainly means to adopt impurity treatment by adopting the technology of microfiltration, ultrafiltration, nanofiltration and inverse filtration in the process of water treatment so as to purify the impurities in the water. This method is not selective. Therefore, useful molecular are lost when the harmful substances are eradicated. However, adopting the MIPs technology in water treatment technology can solve the problem well since MIPs technology can eliminate the pesticide, herbicide, heavy metal, radioactive substance, etc in the water selectively so as to protect the required microelement contained in the water which is needed by human from flowing away. It can be seen that MIPs can gain good development in the field of drinking water treatment.

\section{The Application of Molecular Imprinting Technology in the Sensor}

Sensor is an analytical equipment which possesses selectivity and reversible response and it produces the interactions mainly through the close combination of sensing components and converter. This equipment only responds to the particular matters so that it has selectivity. ${ }^{6}$ Even the biological sensor have extremely high sensitivity and specificity, it's easy for the biologically active components_the components used for identifying the molecular to change, which causes malfunction of the molecular activity. Consequently, the cost of the sensor is increased and the variety of the biologically active components is limited, which results in the limited use of the sensor. In addition, since MIPs have high cross-linking behavior and good stability, so it can be used in extremely severe environment. Therefore, regarding it as the recognization element of the molecular can improve the selectivity and sensitivity, thus further enhancing its tolerance. Moreover, MIPs can not be easily damaged, so it has favorable stability. Therefore, it is favored widely by people.

The sensor made of MIPs can be used in detection areas such as herbicide, saccharides, amino acid and its derivative, solvent, steam, toxin and medicine. Sergeyeva produces imprinting polypropylene acid membrane by considering the atrazine as the template. This membrane can be applied in the sensor which detects atrazine so as to monitor the environment. The monitoring result is $5 \mathrm{nmol} / \mathrm{L}$ and the response time is 6 to 15 minutes. Its detectibility is stable and it can be maintained for 6 months. Jenkins detects the insecticide in water by considering the modified optical of MIPs and optical array as the optical sensor with the detection period being 5 to 10 minutes, response period being 20 to 30m minutes and range of dynamic response linear being $25 * 10^{-7} \sim 1 * 10^{-5}$

\section{The Application of Molecular Imprinting Technology in Enzyme Catalytic Function}

As the cavity inside MIPs is similar to enzyme active center, it possesses certain enzyme catalytic function. Since molecular imprinting can gain certain functional site which can play the role of particular orientation. Therefore, people can obtain the combining site and degeneration genes of the enzyme through molecular imprinting, thus enzyme can further be combined with the substrate, catalyzing the substrate reaction to show the activity of the enzyme. As transient state can promote the generation of the product, regarding the analogue of the transient state as the imprinting can reflect the activity of the enzyme. Compared with natural enzyme, it has the advantage of favorable stability and long service time, so that it is widely used. Molecular imprinting can regulate the activity of the enzyme successfully and produce the mimic enzyme through the catalysis of the natural enzyme.

There were once the scholars who conducted the experiment by using the pyridoxal as the imprinting molecular and manufactured MIPs with 4-vinylcarbazole polymers being the monomer. The substance promotes the proton transfer of the derivative of the amino acid. Then it shows that the application of the polymer of the PVK Polyvinylcarbazole imprinting can enhances the hydrolysis ability of the ester of the high template molecule. Leonhand manufactured the MIPs with 
nitrobenzol-methyl-phosphate being the template and applied the manufactured substrate into the hydrolysis process of nitrobenzol acetate. The conclusion is that MIPs accelerates the hydrolysis process. At present, relevant scholars are hydrolyzing the organic organophosphorous pesticides by taking advantage of the MIPs which contain cobalt and the result is that the MIPs with cobalt have hydrolysis force toward organic organophosphorous pesticides with the hudrolysis rate reaching $24 * 10^{-6} \mathrm{~mol} /(\mathrm{h} \cdot \mathrm{mg})$.

Along with the development of the society, there are more and more synthetic chemical substances. Most substances release harmful materials into the environment, which causes some pollution. However, it is hard to degrade and digest this under the effect of natural enzyme and after a long time, human existence is affected. Degrading the chemical pollutants by utilizing MIPs as catalyst gains highly degradation efficiency so it is widely favored by the environmentalist.

The Application of Molecular Imprinting Technology in Chromatographic Analysis

Under this situation, the number of the theoretical plates for the MIPs column is small, but as MIPs has high selectivity, chiral components can be separated with the electrochromatography of MIPs high efficiency liquid. The chromatography of the template molecule usually manifests as the breadth and hangover of the chromatographic peak, which hampers the development of the technology. Therefore, when applying the MIPs into the capillary electrochromatography technology, the high selectivity of MIPs and the separation efficiency of capillary electrochromatography can be combined to improve the efficiency of the technology. This technology has huge development prospect. In addition, the columner form of the capillary electrochromatography can decrease consumption of the chemical substances of the imprinting molecular so as to improve the work efficiency of MIPs.

Some scholars consider the caffeine as the template molecule and manufactures the capillary monolithic column of molecule imprinting after triggered by UV-light. Then they observe the quality of the monolithic column which influences the molecular imprinting capillary, further optimizing the chromatographic separation conditions. The result shows that the manufactured monolithic column of the molecular imprinting capillary has selectivity toward the caffeine. Therefore, it possesses strong operability when it is applied in the fields such as the detection of green tea beverage, pepsi and compound tablets

\section{Conclusion}

In environmental science, molecular imprinting technology can be carried out in environment detection, sewage treatment, pollutant measurement and environmental modification, so it gains widespread popularity from the environmentalists. Today, with the rapid development of the economy, we should lay more emphasis on the effect of molecular imprinting technology, apply it into the environmental protection and try to make contribution for the environmental protection undertaking in our country.

\section{References}

[1] Lukuan, Zhu Qiujing. The Recognition Mechanism and Evaluation Means based on the Molecular Imprinting Technology of $\beta$ - Cyclodextrin. Functional Material, 2012,43(z1):10-15,21.

[2] Zhaoyan, Lilong, Qinwei. The Study of Bibliometrics based on the Molecular Imprinting Technology of SCI. Malterial Review, 2015,29(5):105-109.

[3] Jiang Yuxiang, Pang Daolin, Heqiang.The Study of identifying the spectrum of the Cephalosporin Molecules with the specificity of Molecular Imprinting Technology. Spectrography and Spectrum Analysis, 2011,31(7):1852-1856.

[4] Tian Rujun, Cheng Shujun, Li Jingna. The Application of Molecular Imprinting Technology in organic matter of Environmental Trace. Sichuan Environment, 2011,30(6):123-127. 
[5] Zhanglu, He Yingying, Wang Xiaochang. The Application of Molecular Imprinting Technology in the residue Separation and Analysis under Antibiotic Environment. Polymer Bulletin, 2013(3):30-35. 